\title{
Increased Neutrophil-To-Lymphocyte Ratio After Thrombolysis Improves Prediction of 3-Month Stroke Outcome
}

\section{Duanlu Hou}

Shanghai fifth people's hospital, Fudan university

\section{Chunjie Wang}

Jiangchuan Community Health Service Center of Minhang District, Shanghai

\section{Xiaofei Ye}

Second Military Medical University

\section{Ping Zhong}

Shanghai Traditional Chinese Medicine Hospital

Danhong Wu ( $\nabla$ danhongwu@fudan.edu.cn)

Shanghai fifth people's hospital, Fudan university

\section{Research Article}

Keywords: neutrophil-to-lymphocyte ratio, acute ischemic stroke, thrombolysis, inflammation, mRS, functional outcome

Posted Date: February 1st, 2021

DOI: https://doi.org/10.21203/rs.3.rs-156684/v1

License: (c) (i) This work is licensed under a Creative Commons Attribution 4.0 International License. Read Full License 


\section{Abstract}

Objectives-Thrombolysis is the effective therapy for acute ischemic stroke (AIS) within 4.5 hours of onset. It improves prognosis by increasing blood perfusion to the lesion. Though inflammation is a predictor of 3-month outcomes after thrombolysis, the temporal effect of thrombolysis on inflammation and the correlation between inflammation after thrombolysis and patients' prognosis remain to be elucidated.

Materials and Methods-Demographic, clinical, laboratory data before and after thrombolysis, and 3month mRS scores were collected and compared. The neutrophil-to-lymphocyte ratio (NLR) was divided into low and high NLR subgroups and functional outcomes at 3 months were compared. Multivariate regression analysis was used to test independent predictors of 3-month prognosis.

Results- 493 patients with AIS were prospectively enrolled between 1st August, 2017 and 1st September, 2019. 170 received thrombolysis and 323 patients did not. The NLR after thrombolysis was higher than that before thrombolysis among patients who received thrombolysis $(p<0.001)$ and higher than that of patients who did not receive thrombolysis $(p<0.001)$. The NLR after thrombolysis was an independent predictor of 3-month functional outcomes ( $\mathrm{OR}=1.20,95 \% \mathrm{Cl}: 1.01-1.42, \mathrm{p}=0.03)$. The best discriminating value for the NLR is 4.51 (sensitivity $65.7 \%$, specificity $73.1 \%$, AUC: $0.85 ; 95 \%$ Cl: $0.77-0.92$ ). Significant difference $(62 \%$ vs $88 \%$, p 0.001$)$ was observed in the patients with good 3-month outcome between patient groups with the NLR $>4.51$ and $<4.51$, respectively.

Conclusions-NLR increase after thrombolysis was a predictor of poor 3-month functional outcome in AIS patients.

\section{Introduction}

Ischemic stroke, a major type of stroke, is a multifactorial disease with high morbidity and mortality $(1,2)$ though two categories of Food and Drug Administration approved therapies are available. Among these therapies, the thrombolytic agent-recombinant tissue plasminogen activator (rt-PA) is the only effective drug for acute ischemic stroke (AIS) within the time frame of 4.5 hours. It can improve the prognosis of AIS patients not only by recanalizing the blood vessel responsible for the ischemic stroke, but also by increasing blood perfusion to the ischemic penumbra. However, the underlying conditions are not cured by rt-PA. Among these conditions, atherosclerosis is the most common one. It is generally acknowledged that atherosclerosis can be exacerbated by inflammation. The latter promotes occurrence of atherosclerosis (3), increases blood vessel pathology (4), and deteriorates blood flow dynamics in ischemia and reperfusion $(5,6)$. Therefore, it is one of the key mechanisms leading to cerebral infarction and influences the prognosis of AIS patients $(3,7)$.

The neutrophil-to-lymphocyte ratio (NLR), an easily acquired, non-invasive, and inexpensive marker of systematic inflammation, has been traditionally treated as an indicator of bacterial infection, showing a more superior predictive value than other conventional inflammatory markers. In addition, it has demonstrated good predictive power for pneumonia (8) as well as the burden of community-acquired 
pneumonia $(8,9)$. It has also been shown that the NLR is a predictor of prognosis for both ischemic and hemorrhagic stroke patients (10-12). In ischemic stroke, the NLR before thrombolysis was proved to be associated with post-thrombolytic functional outcomes $(13,14)$. However, little research has been conducted on the effect of thrombolysis on the inflammatory response of patients with ischemic stroke (15).

The present study aimed to answer this question by investigating the effect of thrombolysis on the NLR of patients with AIS, and subsequently the prognosis of these patients.

\section{Materials And Methods 2.1 Study participants}

Consecutive patients with AIS were screened and recruited from the Acute Ischemic Stroke Registry Program of Shanghai Fifth People's Hospital (The Fifth People's Hospital of Shanghai, Fudan University) between 1st August, 2017 and 1st September, 2019. All recruited patients were followed up in the outpatient to assess their mRS scores at 3 months except those who died or had recurrent stroke during this period. Patients were included if they met the following criteria: (1) diagnosed with AIS within 24 hours of onset; (2) aged $18 \sim 80$ in mainland China; and (3) completed a head magnetic resonance imaging (MRI) within 48 hours. AIS was diagnosed if there were new focal neurological deficits explained by relevant lesions detected on head diffusion-weighted imaging (DWI). Patients were excluded if they met any of the following criteria: (1) presence of cerebral hemorrhage or patients with a history of AIS; (2) pregnant patients; (3) severe heart (with cardiac function in grade $\nabla$ or $\otimes$ according to New York Heart Association or left ventricular ejection fraction $<40 \%$ in echocardiography), lung (with blood oxygen saturation less than $95 \%$ and symptoms of shortness of breath, cyanosis, and abnormal blood gas analysis), liver (serum alanine aminotransferase level $>10$-fold upper limit of the reference range), kidney (serum creatinine $>443 \mu \mathrm{mol} / \mathrm{L}$ ), or neoplastic diseases; (4) autoimmune diseases; (5) symptoms of infection or signs were present at the time of stroke onset. According to the Chinese guideline for AIS management, patients would be treated with intravenous alteplase (dosage $=0.9 \mathrm{mg} / \mathrm{kg} \times$ patient's body weight $(\mathrm{kg}) ; 10 \%$ of the dosage was injected intravenously as a bolus within the first minute and the remaining $90 \%$ injected within 1 hour). The inclusion criteria of thrombolysis were: (1) aged $18 \sim 80$; (2) presented within $4.5 \mathrm{~h}$ after symptom onset, head CT or MRI excluded bleeding lesions; (3) systolic blood pressure $<180 \mathrm{mmHg}$ or diastolic blood pressure $<110 \mathrm{mmHg}$ before treatment; (4) no hemorrhagic disorders or bleeding diathesis; (5) neuro-intervention therapy not considered by patients or their proxies; (6) written informed consent obtained from patients or their families to start rt-PA treatment. AIS patients, aged less than 18 years, with uncertain time of onset, suspected subarachnoid hemorrhage, seizures, intracranial aneurysms, or arteriovenous malformations, or heart, lung, liver, renal insufficiency, or active internal bleeding or arterial puncture within 1 week, surgery within 2 weeks, gastrointestinal or systemic bleeding within 3 weeks, urological trauma within 3 months, or blood glucose $<2.7 \mathrm{mmol} / \mathrm{L}$ or $>22.2$ $\mathrm{mmol} / \mathrm{L}$, were excluded from rt-PA thrombolysis. The flow chart of patient screening and selection was shown in Fig. 1. This study was approved by the Ethical Review Board of Shanghai Fifth People's Hospital 
and written informed consent was obtained from all patients or their families and all methods were performed in accordance with the relevant guidelines and regulations. Baseline data, including age, sex, history of hypertension, diabetes mellitus (DM), atrial fibrillation (AF), dyslipidemia, national institute of health stroke scale (NIHSS) scores, door-to-needle time (DNT), stroke onset-to-needle time (ONT) (stroke onset-to-second blood sampling time), systolic blood pressure (SBP), history of cigarette smoking, and alcohol drinking, were collected from medical records.

\subsection{Laboratory tests}

All blood samples were collected with vacuum tubes, stored at $4{ }^{\circ} \mathrm{C}$, and tested by clinical laboratory technicians within $2 \mathrm{~h}$ after collection. The white blood cell count (WBC), neutrophil proportion (N), lymphocyte proportion $(L)$, platelet count $(P)$, levels of serum total bilirubin, serum direct bilirubin, serum glucose, homocysteine (HCY), triglyceride (TG), total cholesterol (TC), high-density lipoprotein (HDL), and low-density lipoprotein (LDL) were measured. The neutrophil-lymphocyte ratio (NLR) was calculated as the ratio of the neutrophil count to the lymphocyte count $(\mathrm{N} / \mathrm{L})$. These indices were tested for AIS patients who would receive thrombolysis within 1 hour after their presentation to the emergency department and before starting their treatments. For those who did not receive thrombolysis, blood samples were collected only once at the same time as the second blood sample collection for AIS patients who received thrombolysis, that was 6-7am on the second day of admission.

\subsection{Clinical Assessments}

Each enrolled patient underwent a head 3T MRI (Siemens, Forchheim, Germany) scan within 24 hours after AIS onset. AIS patients were divided into 4 subgroups based on the Trial of Org 10172 in Acute Stroke Treatment (TOAST) classification (16): large artery atherosclerosis (LAA), cardio-embolism (CE), small artery occlusion (SAO), and others (including others subtypes). NIHSS scores at admission were used to assess stroke severity and mRS scores at 3 months to assess functional outcomes. The latter was divided into 2 categories: favorable ( $m R S<3$ ) and unfavorable $(m R S \geq 3)$. Minor stroke was defined as NIHSS score $\leq 5$.

\subsection{Statistical analysis}

SPSS 26.0 (IBM Corp., Armonk, NY) and GraphPad Prism 8 (GraphPad Software Inc., La Jolla, CA) were used to perform statistical analysis. Categorical variables were presented as frequency and percentage, and the Pearson's Chi-square test and Fisher's exact test were used to compare between groups. For continuous variables, the Kolmogorov-Smirnov test was used to determine the distribution of data. For data with normal distribution, they were presented as mean $\pm S D$ and the Student $t$ test was used for comparison. For data with abnormal distribution, the median or the interquartile range were presented and the Mann-Whitney $U$ test was used for comparison. Significance was indicated when $p$ value was < 0.05 (two-tailed). Parameters showing a statistical trend $(p<0.1)$ or having been proved to be a significant factor in previous studies were included in the multivariate regression analysis to identify parameters independently associated with functional outcomes. The Hosmer-Lemeshow test was used to test the goodness of fit. The receiver operating characteristic (ROC) curve and Youden's J statistic were 
used to determine the cutoff value of NLR. Then, patients were dichotomized according to the identified cutoff value of NLR, and 3-month mRS scores of different groups divided by the cutoff value of NLR were tested using the Chi-square test and the $z$ test.

\section{Results}

\subsection{Patient characteristics}

Over a period of 2 years, 530 patients with AIS were screened and 37 were excluded based on the exclusion criteria, resulting in 493 patients included in the final analysis. These patients were divided into two groups according to whether they received thrombolysis or not (Table 1). In the thrombolysis group, the average age was $68 \pm 12$ years, $103(60.5 \%)$ were males, $87(51 \%)$ were smokers, and $62(36 \%)$ were drinkers. One hundred and five (61.8\%) patients had hypertension, 28 (16.5\%) diabetes mellitus (DM), 21 $(12.3 \%)$ atrial fibrillation, and $19(11.2 \%)$ history of coronary artery diseases. In the non-thrombolysis group, the average age was $71 \pm 30$ years, 222 (68.7\%) were males, 125 were smokers (39\%), and 69 $(21 \%)$ were drinkers. There was no significant difference in NIHSS scores at admission $(p=0.178)$, SBP $(p=0.754)$, and concentrations of blood glucose $(p=0.873)$ between the two groups. The NLR [ 2.0 (1.53.5) ] was collected and calculated for 164 patients in the thrombolysis group for further comparison. Significant difference was observed in smoking $(p=0.007)$, alcohol drinking $(p<0.001)$, DM $(p=0.001)$, NLR $(p<0.001), \operatorname{TG}(p=0.006)$, levels of LDL $(p=0.003)$ and HDL $(p=0.005)$ between the two groups. These factors along with hypertension, atrial fibrillation, age, sex, and ONT, which were well known risk factors of ischemic stroke, were included in the multivariate regression analysis. Significant difference was observed in NIHSS at 2 weeks $(p<0.001)$ and mRS at 3 months $(p=0.023)$ between the two groups. Regarding the percentage of favorable outcomes (mRS $<3$ ) at 3 months, the thrombolysis group had a lower percentage of patients with favorable outcomes than the non-thrombolysis group $(p=0.020)$. 
Table 1

Baseline characteristics of the study population and bivariate comparisons between thrombolysis and non-thrombolysis patients

$\begin{array}{ll}\text { Thrombolysis } & \text { Non-thrombolysis } \quad p \text { Value } \\ (n=170) & (n=323)\end{array}$

Demographic characteristics

\begin{tabular}{|c|c|c|c|}
\hline Male & $103(60.5)$ & $222(68.7)$ & 0.07 \\
\hline $\mathrm{Age}^{\dagger}$ & $68(12)$ & $69(30)$ & 0.954 \\
\hline Smoker & $87(51)$ & $125(39)$ & $0.007^{*}$ \\
\hline Alcohol drinker & $62(36)$ & $69(21)$ & $<0.001^{*}$ \\
\hline \multicolumn{4}{|l|}{ Medical history } \\
\hline Arterial hypertension & $105(61.8)$ & $216(67)$ & 0.258 \\
\hline Diabetes mellitus & $28(16.5)$ & $96(30)$ & $0.001^{*}$ \\
\hline Atrial fibrillation & $21(12.4)$ & $27(8.4)$ & 0.155 \\
\hline Coronary arterial disease & $19(11.2)$ & $13(4)$ & $0.002^{*}$ \\
\hline \multicolumn{4}{|l|}{ Clinical characteristics } \\
\hline Admission NIHSS scores ${ }^{\ddagger}$ & $5(3-10)$ & $4(2-6)$ & 0.178 \\
\hline Admission systolic BP, mmHg ${ }^{\ddagger}$ & $180(160-190)$ & $180(160-194)$ & 0.754 \\
\hline Blood glucose, $\mathrm{mg} / \mathrm{dl}^{\ddagger}$ & $5.4(4.7-6.9)$ & $5.5(4.7-7.2)$ & 0.873 \\
\hline \multicolumn{4}{|l|}{ Laboratory data } \\
\hline Neutrophil-lymphocyte ratio ${ }^{\ddagger}$ & $3.5(2.3-5.6)$ & $2.7(1.9-3.9)$ & $<0.001^{*}$ \\
\hline Platelet, $10^{9} / \mathrm{L}$ & $199(170-236)$ & $205(165-241)$ & 0.681 \\
\hline
\end{tabular}

Abbreviations: NIHSS $=$ National institutes of health stroke scale; $\mathrm{BP}=$ blood pressure; $\mathrm{LDL}=$ low density lipoprotein; $\mathrm{HDL}$ = high density lipoprotein; $\mathrm{TG}$ = total cholesterol; $\mathrm{mRS}$ = modified Rankin scale.

Unless specified, values are numbers of patients (\%).

${ }^{\dagger}$ Mean (standard deviation).

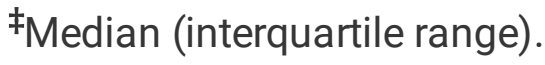

*Statistically significant. 


\begin{tabular}{|c|c|c|c|}
\hline & $\begin{array}{l}\text { Thrombolysis } \\
(n=170)\end{array}$ & $\begin{array}{l}\text { Non-thrombolysis } \\
(n=323)\end{array}$ & $p$ Value \\
\hline $\mathrm{LDL}, \mathrm{mmol} / \mathrm{L}^{\ddagger}$ & $2.9(2.4-3.5)$ & $2.7(2.1-3.3)$ & $0.003^{*}$ \\
\hline $\mathrm{HDL}, \mathrm{mmol} / \mathrm{L}^{\ddagger}$ & $1.1(0.9-1.3)$ & $1(0.9-1.3)$ & $0.005^{\star}$ \\
\hline $\mathrm{TG}, \mathrm{mmol} / \mathrm{L}^{\ddagger}$ & $4.5(3.7-4.9)$ & $4.1(3.5-4.8)$ & $0.006^{*}$ \\
\hline Triglyceride, $\mathrm{mmol} / \mathrm{L}^{\ddagger}$ & $1.2(0.9-1.7)$ & $1.3(0.9-1.9)$ & 0.985 \\
\hline Total bilirubin, $\mu \mathrm{mol} / \mathrm{L}^{\ddagger}$ & $12(9.2-17.0)$ & $11.3(8.4-14.8)$ & 0.172 \\
\hline Homocysteine, $\mu \mathrm{mol} / \mathrm{L}^{\ddagger}$ & $13.3(10.4-18.8)$ & $14.4(11.4-17.9)$ & 0.122 \\
\hline \multicolumn{4}{|l|}{ Medication (rtPA or routine) } \\
\hline Door-to-needle time, min $^{\ddagger}$ & $74(55-103)$ & - & - \\
\hline Onset-to-needle time, $\mathrm{h}^{\ddagger}$ & $14(13-15)$ & $24(15-48)$ & $<0.0001^{*}$ \\
\hline \multicolumn{4}{|l|}{ Outcomes } \\
\hline 2-week NIHSS ${ }^{\ddagger}$ & $2(1-5)$ & $1(1-3)$ & $<0.001^{*}$ \\
\hline 3-month mRS & $1(0-3)$ & $1(0-2)$ & $0.023^{*}$ \\
\hline Favorable outcome (mRS < 3 ) & $120(71)$ & $258(79)$ & $0.020^{\star}$ \\
\hline Unfavorable outcome (mRS > = 3 ) & $50(29)$ & $65(21)$ & - \\
\hline \multicolumn{4}{|c|}{$\begin{array}{l}\text { Abbreviations: NIHSS = National institutes of health stroke scale; } B P=\text { blood pressure; } L D L=\text { low } \\
\text { density lipoprotein; } \mathrm{HDL}=\text { high density lipoprotein; } \mathrm{TG}=\text { total cholesterol; } \mathrm{mRS}=\text { modified Rankin } \\
\text { scale. }\end{array}$} \\
\hline \multicolumn{4}{|c|}{ Unless specified, values are numbers of patients (\%). } \\
\hline \multicolumn{4}{|l|}{${ }^{\dagger}$ Mean (standard deviation). } \\
\hline \multicolumn{4}{|l|}{${ }^{\ddagger}$ Median (interquartile range). } \\
\hline *Statistically significant. & & & \\
\hline
\end{tabular}

In patients receiving thrombolysis, variables including total bilirubin, NLR, and NIHSS were compared before and after thrombolysis (Table 2$)$. The NIHSS was significantly decreased $(p<0.001)$ and NLR significantly increased $(p<0.001)$ after thrombolysis. No significant difference was observed in concentrations of serum total bilirubin $(p=0.069)$. Intracerebral bleeding due to thrombolysis was not found in the first two days after thrombolysis. 
Table 2

Comparisons of factor changes between before and after medication in thrombolysis

\begin{tabular}{|llll}
\hline & Before thrombolysis & After thrombolysis & $p$ Value \\
\hline TBil & $13.4(9.8-17.7)$ & $12.1(9.2-17)$ & 0.0692 \\
\hline NLR & $2.0(1.5-3.5)$ & $3.5(2.3-5.6)$ & $<0.001^{*}$ \\
\hline NIHSS & $5(3-10)$ & $4(1-7)$ & $<0.001^{*}$ \\
\hline
\end{tabular}

Abbreviations: TBil = total bilirubin; NLR = neutrophil-lymphocyte ratio; NIHSS = National institutes of health stroke scale.

Unless specified, values are medians (interquartile range).

*Statistically significant.

\subsection{Association between NLR and functional outcomes}

ROC analysis identified 4.51 as the best cutoff value of NLR to discriminate between favorable and unfavorable functional outcomes after thrombolysis at 3 months (blue line, area under the curve-AUC = 0.85, 95\%Cl: 0.77-0.92; $p<0.001$; Youden's index $=0.388$; sensitivity: $65.7 \%$, specificity: $73.1 \%$; Fig. 2). Additional ROC analysis also showed independent associations of NLR before thrombolysis (red line, AUC $=0.75, p<0.001$, Youden's index $=0.222$; sensitivity: $87.6 \%$, specificity: $34.6 \%$; Fig. 2 ) and NLR without thrombolysis (green line, AUC $=0.63, p<0.001$, Youden's index $=0.265$; sensitivity: $58.3 \%$, specificity: 68.2\%; Fig. 2) with functional outcomes at 3 months.

Based on the best cut-off value of the ROC curve, patients receiving and not receiving thrombolysis were divided into two groups - the high NLR and the low NLR groups, respectively. Proportions of patients with good 3-month functional outcomes were compared between these two groups (Table 3). It was found that the proportion of patients with good prognosis at 3 months in the high NLR group was significantly smaller than that in the low NLR group $(p=0.006)$ for patients receiving thrombolysis. No significant difference was observed in this proportion between the two groups for patients not receiving thrombolysis. 
Table 3

Comparisons of favorable outcome based on cutoff value of neutrophil-lymphocyte ratio

\begin{tabular}{|llll|}
\hline & Low NLR & Higher NLR & p \\
\hline Thrombolysis & $72(80)$ & $22(44)$ & $0.0001^{*}$ \\
\hline Non-thrombolysis & $205(66)$ & $34(70)$ & 0.097 \\
\hline & Unless specified, values are numbers of patients (\%). \\
\hline
\end{tabular}

\subsection{Multivariable regression analysis for confounders with favorable functional outcome}

After comparing the potential factors such as age, sex, ONT, hypertension, diabetes mellitus, atrial fibrillation, alcohol drinking, levels of HDL and LDL, and NLR after thrombolysis (admission NLR) (Table 4), it was found that NLR after thrombolysis could independently predict functional outcomes at 3 months $(\mathrm{OR}=1.20,95 \% \mathrm{Cl}, 1.01-1.42, p=0.03)$. Alcohol drinking was also found to be an independent risk factor of functional outcomes at 3 months. The other factors did not show such predicting power.

Table 4. Multivariable regression analysis for risk factors with favorable functional outcome

\begin{tabular}{|llllll|}
\hline & Beta & SE & Wald & $\boldsymbol{p}$ & OR, 95\% Cl \\
\hline Age & 0.043 & 0.028 & 2.293 & 0.13 & $1.04,0.99-1.10$ \\
\hline ONT, h & 0.495 & 0.687 & 0.520 & 0.47 & $1.64,0.43-6.3$ \\
\hline Hypertension & -0.07 & 0.175 & 0.161 & 0.69 & $0.93,0.66-1.31$ \\
\hline Diabetes & -0.729 & 0.600 & 1.476 & 0.22 & $0.48,0.15-1.56$ \\
\hline Atrial fibrillation & 0.434 & 0.755 & 0.331 & 0.57 & $1.54,0.35-6.78$ \\
\hline Alcohol drinker & 2.864 & 0.805 & 12.633 & $<0.001^{*}$ & $17.5,3.6-84.9$ \\
\hline TG, mmol/L & -1.251 & 0.648 & 3.731 & 0.053 & $0.29,0.08-1.02$ \\
\hline Admission NLR ${ }^{\dagger}$ & 0.200 & 0.086 & 4.356 & $0.03^{*}$ & $1.20,1.01-1.42$ \\
\hline HDL, mmol/L & -0.197 & 0.830 & 0.056 & 0.81 & $0.82,0.16-4.18$ \\
\hline LDL, mmol/L & -0.006 & 0.023 & 0.064 & 0.80 & $0.99,0.95-1.04$ \\
\hline Constant & -3.675 & 3.594 & 1.046 & 0.31 & - \\
\hline
\end{tabular}


Abbreviations: $\mathrm{SE}=$ standard error; $\mathrm{OR}=$ odd ratio; $\mathrm{Cl}$ = confidence interval; ONT = onset-to-needle time; $\mathrm{TG}$ = total cholesterol; NLR = neutrophil-lymphocyte ratio; $\mathrm{HDL}$ = high density lipoprotein; $\mathrm{LDL}=$ low density lipoprotein.

${ }^{\dagger} \mathrm{NLR}$ after thrombolysis.

*Statistically significant.

\section{Discussion}

In this single-center prospective cohort study, we found that the NLR can significantly increase after thrombolysis, and this increased NLR can predict adverse outcomes at 3 months, suggesting that thrombolysis might aggravate inflammatory injury to the brain and impact the prognosis of patients with AIS.

We found that the NLR level of patients receiving thrombolysis at admission (that is after thrombolysis) was significantly higher than that of patients who did not receive thrombolysis (Table 1). Similarly, the NLR level of patients receiving thrombolysis at admission (after thrombolysis) was significantly higher than that before thrombolysis (Table 2) and this NLR after thrombolysis could independently predict adverse outcomes in the multivariate regression analysis (Table 4). These suggest that thrombolysis could increase the NLR.

In the present study, the majority of patients in the 2 groups were hypertensive with a median systolic blood pressure of $180 \mathrm{mmHg}$ as measured in the Neurology Ward. As stated in the Chinese guideline for thrombolytic therapy, patients with a systolic blood pressure greater than $180 \mathrm{mmHg}$ or blood glucose less than $2.7 \mathrm{mmol} / \mathrm{L}$ or greater than $22.2 \mathrm{mmol} / \mathrm{L}$ are not recommended for thrombolysis. When a patient's blood pressure at presentation is greater than $180 \mathrm{mmHg}$, the clinician will decide whether this patient should receive thrombolysis after controlling the blood pressure with antihypertensives by taking other factors into account. If confirmed, patients will be closely monitored with electrocardiography and the thrombolytic agent will be intravenously injected when the blood pressure drops. In the present study, none of the patients in the thrombolysis group had blood glucose lower than $2.7 \mathrm{mmol} / \mathrm{L}$ or greater than $22.2 \mathrm{mmol} / \mathrm{L}$, therefore, none of them had blood glucose-related contraindications for thrombolysis.

The underlying mechanism of NLR on stroke is due to the central role neutrophils play in all types of stroke. Circulating neutrophils are the first among various peripheral inflammatory cells derived from the bone marrow and spleen to infiltrate the ischemic lesion (30 min to a few hours)(17). This infiltration peaks $24-72 \mathrm{~h}$ after the onset of ischemic stroke and decreases rapidly afterwards, which is associated with stroke severity (18), infarct size (19), and worsened neurological functions (20). In the first 15 minutes of infiltration, neutrophils express endothelial cell adhesion molecules, P-selection glycoprotein ligand-1, lymphocyte function-associated antigen 1, and macrophage-1 antigen (21). By 2 hours, they begin to roll and adhere to pial vessels of the brain, interacting with the endothelial cells. By $6-8$ hours, extravasation of neutrophils starts (22) and peaks at 24-72 hours (23). Adhesion of neutrophils activates 
the endothelium through adhesion molecules disrupting the integrity of the blood brain barrier. As a result, brain edema occurs (24), aggravating stroke severity and deteriorating stroke outcomes (25).

In contrast to neutrophils, lymphocytes decrease in number after ischemic stroke (24). Lymphocytes, especially $C D 4+, C D 8+T$ cells and $\gamma \delta T$ cells, play important roles in inflammation by producing proinflammatory cytokines such as interferon-y and IL-17 (26). In contrast, Treg cells (CD4 + CD25 + Foxp3 + $T$ cell) attenuate inflammation by releasing anti-inflammatory cytokines such as IL-10 which protects the brain through the IL-10/JAK/STAT, PI3K, and MAPK pathways $(25,27)$. The decrease in the number of lymphocytes and increase in the number of neutrophils leads to increased NLR after stroke. Furthermore, an elevated NLR negatively impacts the functional outcome of patients with AIS due to secondary brain injury induced by neutrophil activation and due to increased risk of infection by lymphocyte suppression. After successful recanalization of large occluded vessels, the NLR falls with reperfusion of ischemic lesions (28). It has been reported that the NLR decreases 72 hours after successful recanalization (28). However, an elevated neutrophil count has been considered an independent predictor of poor outcome $(\mathrm{mRS}>3$ ) at 90 days despite $\mathrm{TICl} 2 \mathrm{~b} / 3$ recanalization (28). A study has reported that increased NLR after thrombolysis is independently correlated with symptomatic intracerebral hemorrhage and poor functional outcomes at 3 months (11).

The present study found that the NLR was increased after intravenous thrombolysis and this elevated NLR was an independent predictor of unfavorable outcomes. This suggests that thrombolysis-mediated inflammatory response is likely to be attributed to the increased number of neutrophils or decreased number of lymphocytes. Recanalization of blood vessels by thrombolysis potentially promotes the release of early reactive cells, such as neutrophils, which infiltrate into the ischemic lesion through the disrupted blood-brain barrier to damage the ischemic cells in the penumbra. To remove these damaged cells, lymphocytes are recruited and exhausted. As a result, the number of lymphocytes was decreased. This mechanism was tentatively coined as a second hit by inflammatory flood. For patients with successful recanalization after thrombolysis, this seems to be an inevitable process. This is why the elevated NLR has been widely reported to be associated with poor prognosis in cardiovascular studies.

There are a number of limitations in the present study. Firstly, the present cohort study was conducted in a single stroke center and only recruited the Chinese population. Patients receiving thrombolysis were not randomly included, which might lead to selection bias. Secondly, the NLR was only tested at admission without following its temporal changes, which might impact the correlation between NLR and progression of ischemic stroke. Thirdly, only NIHSS and mRS scores were used to evaluate functional outcomes of stroke patients. No other measures, such as advanced cognitive function, were used, which might compromise our conclusion.

\section{Conclusions}

Thrombolytic therapy increases the NLR of patients with AIS, and this increased NLR predicts poor functional outcomes at 3 months. Therefore, reperfusion after thrombolysis leads to secondary injury to 
the existing lesion by aggravating inflammation.

\section{Abbreviations}

AIS, acute ischemic stroke; mRS, modified Rankin scale; NLR, neutrophil-to-lymphocyte ratio; OR, odd ratio; $\mathrm{Cl}$, confidence interval; AUC, area under curve; FDA, Food and Drug Administration; rt-PA, recombinant tissue plasminogen activator; MRI, magnetic resonance imaging; DWI, diffusion-weighted imaging; DM, diabetes mellitus; AF, atrial fibrillation; NIHSS, national institute of health stroke scale; DNT, door-to-needle time; ONT, stroke onset-to-needle time/(stroke onset-to-second blood sampling time); SBP, systolic blood pressure; WBC, white blood cell count; N, neutrophil proportion; L, lymphocyte proportion; $\mathrm{P}$, platelet count; $\mathrm{HCY}$, homocysteine; TG, triglyceride; TC, total cholesterol; HDL, high-density lipoprotein; LDL, low-density lipoprotein; TOAST, Trial of Org 10172 in Acute Stroke Treatment; LAA, large artery atherosclerosis; $\mathrm{CE}$, cardio-embolism; SAO, small artery occlusion; ROC, receiver operating characteristic.

\section{Declarations}

\section{Acknowledgements}

Not applicable

\section{Funding}

This work was supported by the Shanghai Committee of Science and Technology (Grant No. 201409004900), and Shanghai Fifth People's Hospital, Fudan university (Grant No. 2018WYZD01).

\section{Authors' contributions}

DW and DH designed this project. DH and CW collected data. DH and $\mathrm{XY}$ analyzed data, and $\mathrm{DH}$ drafted the manuscript. DW and DH evaluated MRI results. DW and PZ proofread and reviewed the manuscript. $\mathrm{DH}$ polished the final manuscript. All authors read and approved the final manuscript.

\section{Declaration of interest statement}

The authors declare that they have no competing interests.

\section{References}

1. Shroff N, Ander BP, Zhan X, Stamova B, Liu D, Hull H, et al. HDAC9 Polymorphism Alters Blood Gene Expression in Patients with Large Vessel Atherosclerotic Stroke. Transl Stroke Res. 2019;10(1):19-25. 2. Hankey GJ. Stroke. Lancet (London, England). 2017;389(10069):641-54.

3. Wolf D, Ley K. Immunity and Inflammation in Atherosclerosis. Circulation research. 2019;124(2):31527. 
4. Murray KN, Girard S, Holmes WM, Parkes LM, Williams SR, Parry-Jones AR, et al. Systemic inflammation impairs tissue reperfusion through endothelin-dependent mechanisms in cerebral ischemia. Stroke. 2014;45(11):3412-9.

5. Guo ZN, Guo WT, Liu J, Chang J, Ma H, Zhang P, et al. Changes in cerebral autoregulation and blood biomarkers after remote ischemic preconditioning. Neurology. 2019;93(1):e8-e19.

6. Burrows F, Haley MJ, Scott E, Coutts G, Lawrence CB, Allan SM, et al. Systemic inflammation affects reperfusion following transient cerebral ischaemia. Exp Neurol. 2016;277:252-60.

7. Esenwa CC, Elkind MS. Inflammatory risk factors, biomarkers and associated therapy in ischaemic stroke. Nat Rev Neurol. 2016;12(10):594-604.

8. Curbelo J, Rajas O, Arnalich B, Galvan-Roman JM, Luquero-Bueno S, Ortega-Gomez M, et al. Neutrophil Count Percentage and Neutrophil-Lymphocyte Ratio as Prognostic Markers in Patients Hospitalized for Community-Acquired Pneumonia. Arch Bronconeumol. 2019;55(9):472-7.

9. Nam KW, Kim TJ, Lee JS, Kwon HM, Lee YS, Ko SB, et al. High Neutrophil-to-Lymphocyte Ratio Predicts Stroke-Associated Pneumonia. Stroke. 2018;49(8):1886-92.

10. Zhu B, Pan Y, Jing J, Meng X, Zhao X, Liu L, et al. Neutrophil counts, neutrophil ratio, and new stroke in minor ischemic stroke or TIA. Neurology. 2018;90(21):e1870-e8.

11. Lattanzi S, Cagnetti C, Provinciali L, Silvestrini M. Neutrophil-to-Lymphocyte Ratio Predicts the Outcome of Acute Intracerebral Hemorrhage. Stroke. 2016;47(6):1654-7.

12. Pikija S, Sztriha LK, Killer-Oberpfalzer M, Weymayr F, Hecker C, Ramesmayer C, et al. Neutrophil to lymphocyte ratio predicts intracranial hemorrhage after endovascular thrombectomy in acute ischemic stroke. J Neuroinflammation. 2018;15(1):319.

13. Maestrini I, Strbian D, Gautier S, Haapaniemi E, Moulin S, Sairanen T, et al. Higher neutrophil counts before thrombolysis for cerebral ischemia predict worse outcomes. Neurology. 2015;85(16):1408-16.

14. Liu H, Wang R, Shi J, Zhang Y, Huang Z, You S, et al. Baseline Neutrophil Counts and Neutrophil Ratio May Predict a Poor Clinical Outcome in Minor Stroke Patients with intravenous Thrombolysis. Journal of stroke and cerebrovascular diseases : the official journal of National Stroke Association. 2019;28(11):104340.

15. Guo Z, Yu S, Xiao L, Chen X, Ye R, Zheng P, et al. Dynamic change of neutrophil to lymphocyte ratio and hemorrhagic transformation after thrombolysis in stroke. J Neuroinflammation. 2016;13(1):199.

16. Adams HP, Jr., Bendixen BH, Kappelle LJ, Biller J, Love BB, Gordon DL, et al. Classification of subtype of acute ischemic stroke. Definitions for use in a multicenter clinical trial. TOAST. Trial of Org 10172 in Acute Stroke Treatment. Stroke. 1993;24(1):35-41.

17. Ross AM, Hurn P, Perrin N, Wood L, Carlini W, Potempa K. Evidence of the peripheral inflammatory response in patients with transient ischemic attack. Journal of stroke and cerebrovascular diseases : the official journal of National Stroke Association. 2007;16(5):203-7.

18. Kim J, Song TJ, Park JH, Lee HS, Nam CM, Nam HS, et al. Different prognostic value of white blood cell subtypes in patients with acute cerebral infarction. Atherosclerosis. 2012;222(2):464-7. 
19. Buck BH, Liebeskind DS, Saver JL, Bang OY, Yun SW, Starkman S, et al. Early neutrophilia is associated with volume of ischemic tissue in acute stroke. Stroke. 2008;39(2):355-60.

20. Kumar AD, Boehme AK, Siegler JE, Gillette M, Albright KC, Martin-Schild S. Leukocytosis in patients with neurologic deterioration after acute ischemic stroke is associated with poor outcomes. Journal of stroke and cerebrovascular diseases : the official journal of National Stroke Association. 2013;22(7):e111-7.

21. Kataoka H, Kim SW, Plesnila N. Leukocyte-endothelium interactions during permanent focal cerebral ischemia in mice. Journal of cerebral blood flow and metabolism : official journal of the International Society of Cerebral Blood Flow and Metabolism. 2004;24(6):668-76.

22. Watcharotayangul J, Mao L, Xu H, Vetri F, Baughman VL, Paisansathan C, et al. Post-ischemic vascular adhesion protein-1 inhibition provides neuroprotection in a rat temporary middle cerebral artery occlusion model. J Neurochem. 2012;123 Suppl 2:116-24.

23. Tu XK, Yang WZ, Shi SS, Wang CH, Zhang GL, Ni TR, et al. Spatio-temporal distribution of inflammatory reaction and expression of TLR2/4 signaling pathway in rat brain following permanent focal cerebral ischemia. Neurochem Res. 2010;35(8):1147-55.

24. Jickling GC, Liu D, Ander BP, Stamova B, Zhan X, Sharp FR. Targeting neutrophils in ischemic stroke: translational insights from experimental studies. Journal of cerebral blood flow and metabolism : official journal of the International Society of Cerebral Blood Flow and Metabolism. 2015;35(6):888901.

25. Akopov SE, Simonian NA, Grigorian GS. Dynamics of polymorphonuclear leukocyte accumulation in acute cerebral infarction and their correlation with brain tissue damage. Stroke. 1996;27(10):1739-43.

26. Yilmaz G, Arumugam TV, Stokes KY, Granger DN. Role of T lymphocytes and interferon-gamma in ischemic stroke. Circulation. 2006;113(17):2105-12.

27. Ooboshi H, Ibayashi S, Shichita T, Kumai Y, Takada J, Ago T, et al. Postischemic gene transfer of interleukin-10 protects against both focal and global brain ischemia. Circulation. 2005;111(7):913-9.

28. Goyal N, Tsivgoulis G, Chang JJ, Malhotra K, Pandhi A, Ishfaq MF, et al. Admission Neutrophil-toLymphocyte Ratio as a Prognostic Biomarker of Outcomes in Large Vessel Occlusion Strokes. Stroke. 2018;49(8):1985-7.

\section{Figures}




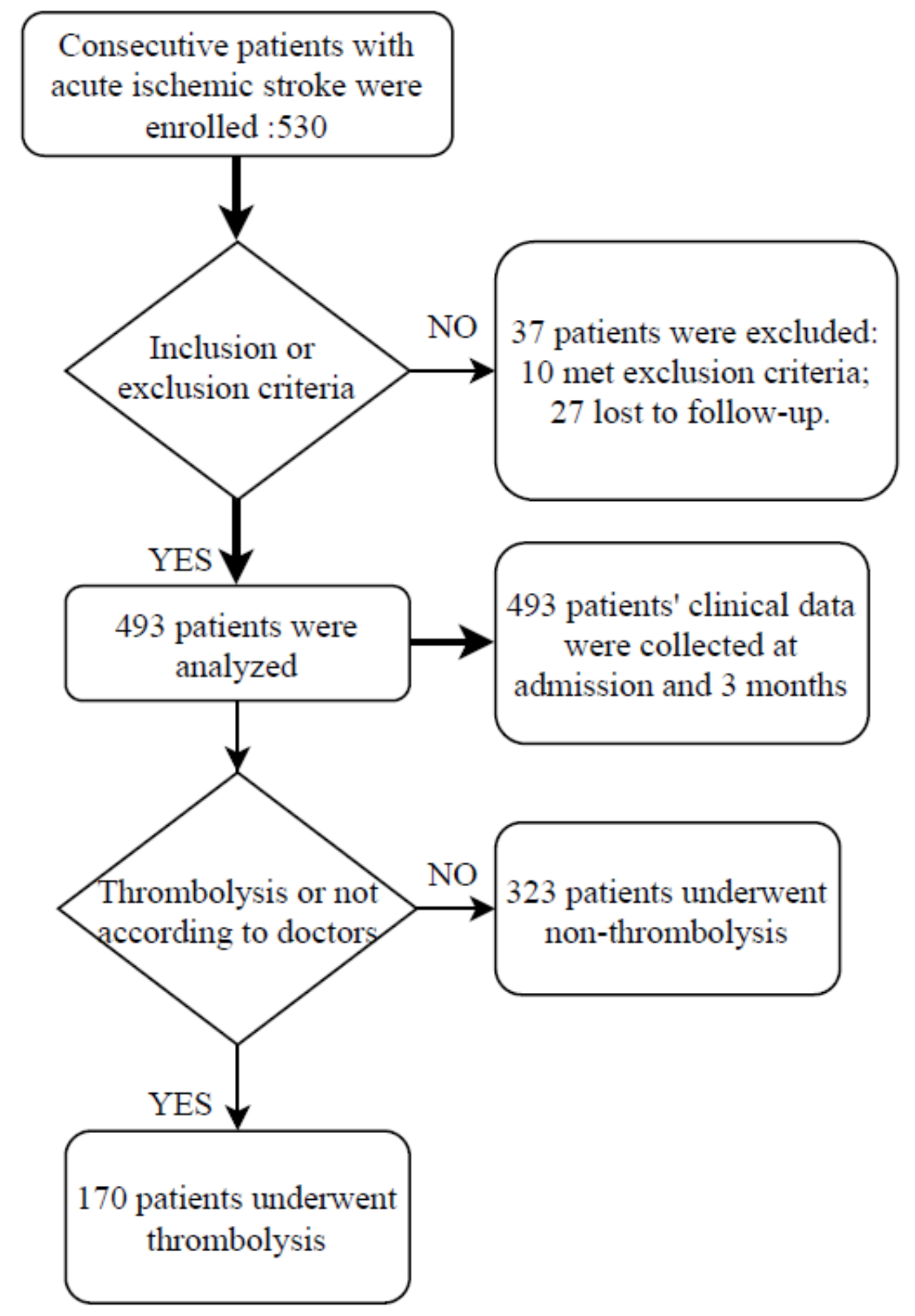

Figure 1

Flowchart of patients enrollment and follow-up 


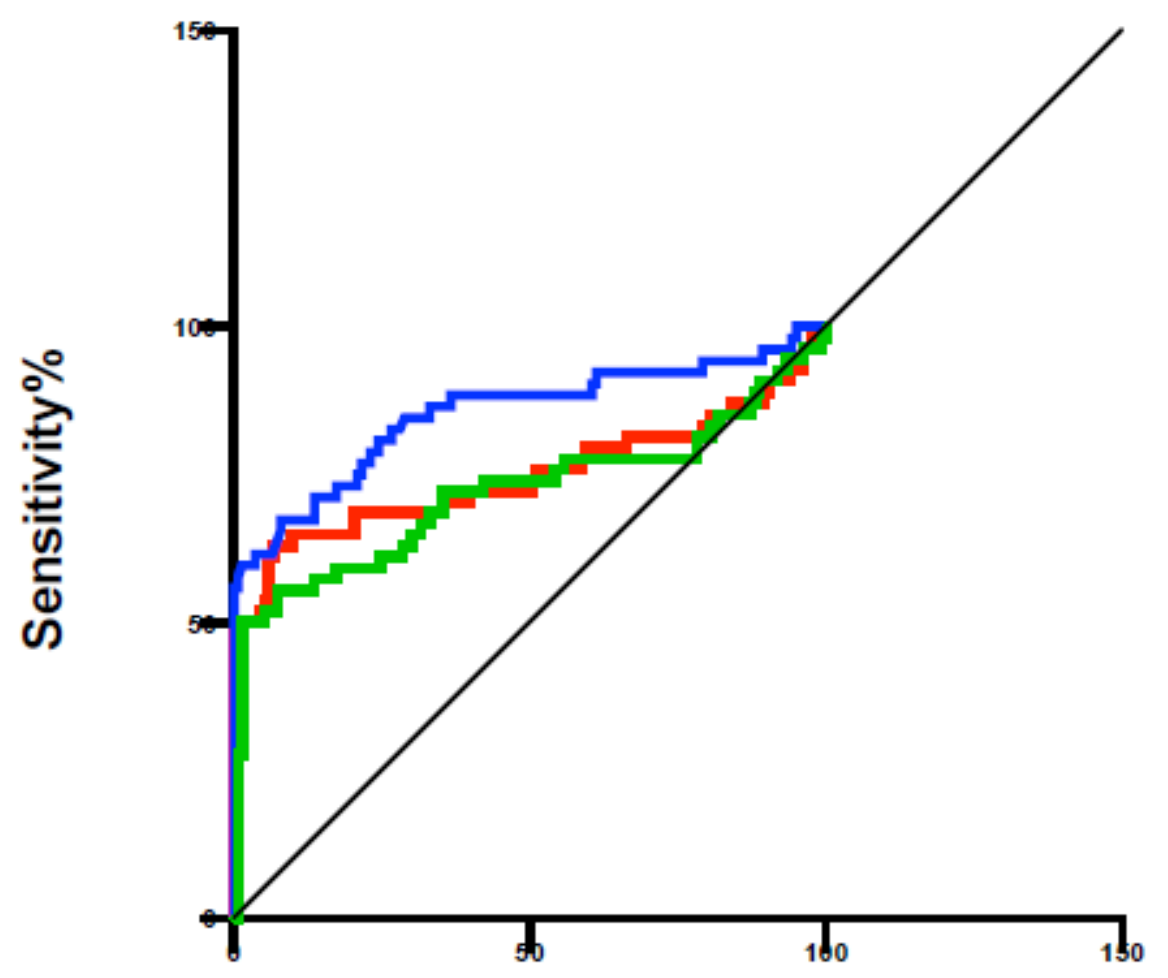

\section{$100 \%$ - Specificity $\%$}

Figure 2

Receiver operating characteristic (ROC) curve for neutrophil-lymphocyte ratio (NLR) to predict 3-month post-thrombolytic outcome 